\title{
Constraint Sparse Maximum Correntropy Beamforming Algorithm against Impulsive Noise to Improve the Performance of GNSS Signal Acquisition
}

\author{
Haichuan Zhang $(\mathbb{D})$ and Fangling Zeng \\ School of Electronic Countermeasures, National University of Defense Technology, Hefei 230037, China \\ Correspondence should be addressed to Haichuan Zhang; zhanghai4258@163.com
}

Received 11 March 2019; Revised 25 April 2019; Accepted 8 May 2019; Published 18 June 2019

Academic Editor: Giorgio Montisci

Copyright (C) 2019 Haichuan Zhang and Fangling Zeng. This is an open access article distributed under the Creative Commons Attribution License, which permits unrestricted use, distribution, and reproduction in any medium, provided the original work is properly cited.

\begin{abstract}
This paper presents the new constraint correntropy beamforming method to improve the performance of global navigation satellite system (GNSS) receivers. Although beamforming-based GNSS system possesses the ability of nulling interference sources, the distortions to satellite signal induced by impulsive noises are always neglected. This paper addresses the satellite navigation signal acquisition problem against impulsive noises using the constraint maximum correntropy criterion in framework of the GNSS system. In addition, in order to decrease the number of active elements for avoiding overmuch energy consumption, we introduce the L1-norm penalty equation to the list of constraints of the adaptive filter that forces the coefficients with small magnitudes to zero. From the above, we propose the norm-constraint beamforming method to satisfy the conflicting requirements between the coefficients sparsity and the performance of the satellite signal acquisition. The proposed CSMC maintains the robustness against impulsive outliers and achieve better performance in conjunction with less power consumption. An analysis of the mean square convergence properties of the proposed algorithms is presented, and the stability condition of the convergence is derived. Our simulation results demonstrate the superiority of the proposed methods.
\end{abstract}

\section{Introduction}

Adaptive beamforming, as one of the most important techniques of array signal processing has been widely used in GNSS system as well as other applications because of its efficient ability to suppress interference and preserve desired signal $[1,2]$. The GNSS signal is collected by receiver in open sky conditions, and the power of satellite signal on the ground is very weak [3]; thus, the GNSS receivers are susceptible to intentional or unintentional jamming. Moreover, the navigation performance of the receiver mostly depends on the process of satellite signal acquisition [4], and the theoretically predicted performance of GNSS system in practical application may not be achieved due to various factors including impulsive noises $[5,6]$. Consequently, adaptive beamforming criterions which favor GNSS system and could also mitigate the effects of these imperfections are motivated into research including time-domain, transform-domain, and space-domain algorithms [7-9], while time-domain and transform-domain techniques can hardly be used to inhibit broadband interferences, and the spatial interference suppression based on the adaptive beamforming has been considered to be a promising solution to this problem. The algorithms proposed in $[10,11]$ have opened a way for the beamformer processing to play an important role in GNSS receivers; the effectiveness of these beamforming methods for suppressing interferences and enhancing satellite signals has been studied and verified in the papers. In the other open literature, Zhou designed a jointly adaptive beamforming method based on maximum signal-to-noise ratio (MSNR) criteria to suppress the jammers [12]; this technique is unable to track the interference rapidly for its relatively high computation complexity. Classical LMS (least mean square) family algorithms such as Normalized Least Mean Square (NLMS) [13, 14], Constrained LMS (CLMS) [15, 16], and modified robust variable step size (MRVSS) [17] are the 
popular beamforming methods since they can achieve fast convergence for real-time communication without causing too much performance degradation. However, the criterion of these algorithms utilized exhibits performance degradation in the presence of alpha noise, particularly on the process of satellite signal acquisition. Therefore, it is necessary to develop a robust beamforming method against impulsive noises with application to GNSS receiver.

Maximum correntropy criterion (MCC) has gained a wide attention for its simplicity and insensitivity to outliers, MCC-based methods have been widely applied to principal component analysis [18], system identification [19], and other related fields in recent years [20]. However, this technique rarely has been applied to the beamforming and GNSS field. Besides, most beamforming algorithms suppress the interference effectively with high power consumption; as a result, the applicability of these techniques is restricted by the scenarios with limited power supply systems. With considerations on these issues, the main contributions of this paper are summarized as follows: we propose the novel adaptive beamforming algorithms in the presence of impulsive noise, referred to as constrained sparse maximum correntropy. The novel algorithm takes into account the possibility for provoking sparsity of L1-norm and adds a particular norm constraint into the optimization function. The results of this technique implementation can be regarded as producing the sparse solution of adaptive beamformer and achieving less waste of power at the GNSS receiver. Besides, we exploit the prior knowledge that MCC is robust against impulsive process of external perturbation; the optimization problem in mean square error (MSE) sense is transferred into the maximization of MCC function to attenuate the adverse effects of impulsive noises. Thus, the proposed CSMC beamforming technique can achieve large degrees of sparsity of the beamformer array at GNSS receiver while maintaining the robustness of MCC against impulsive noises.

The structure of the paper is organized as follows: satellite navigation signal reception model of GNSS receiver is presented in Section 2. Section 3 presents the proposed robust CSMC beamforming method and provides the mean square analysis of the algorithm. Section 4 illustrates and analyzes the simulation results. Section 5 summarizes the conclusions.

\section{Satellite Navigation Signal Reception Model}

Consider a uniform linear antenna array of $M$ omnidirectional array elements employed in GNSS receiver, one satellite signal, and $Q$ uncorrelated interference which impinge on the array at the $k$ th snapshot as expressed by [21]

$$
\mathbf{x}(k)=s(k) \mathbf{a}\left(\theta_{d}\right)+\sum_{i=1}^{Q} i_{i}(k) \mathbf{a}\left(\theta_{i}\right)+\mathbf{n}(k)
$$

where $s(k)$ and $i_{i}(k)$ are the desired GNSS signal and the $i$ th interference and $\mathbf{n}(k)$ is the noise vector. $\mathbf{a}\left(\theta_{d}\right)$ and $\mathbf{a}\left(\theta_{i}\right)$ represent $M \times 1$ steering vectors of $s(k)$ and $i_{i}(k)$ as given by

$$
\begin{aligned}
\mathbf{a}\left(\theta_{d}\right) & =\left[1, e^{-j 2 \pi\left(d_{c} / \lambda\right) \cos \theta_{d}}, \ldots, e^{-j 2 \pi\left(d_{c} / \lambda\right)(M-1) \cos \theta_{d}}\right]^{T} \\
\mathbf{a}\left(\theta_{i}\right) & =\left[1, e^{-j 2 \pi\left(d_{c} / \lambda\right) \cos \theta_{i}}, \ldots, e^{-j 2 \pi\left(d_{c} / \lambda\right)(M-1) \cos \theta_{i}}\right]^{T}
\end{aligned}
$$

where $\theta_{d}$ and $\theta_{i}$ denote the direction of GNSS signal and the $i$ th interference, $d_{c}$ is the interelement spacing, $\lambda$ is the wavelength of GNSS carrier, and $T$ is the transpose operation. The GNSS signal $s(k)$ at L1 band can be written as

$$
s(k)=\sqrt{\mathrm{E}} c_{\mathrm{C} / \mathrm{A}}(k) d_{\mathrm{N} / \mathrm{A}}(k) \sin \left(2 \pi f_{\mathrm{L} 1} k+\theta_{\mathrm{L} 1}\right)
$$

where $\mathrm{E}$ is power of the receiving GNSS signal, $d_{\mathrm{N} / \mathrm{A}}(k)$ represents the data code carrying navigational message, $c_{\mathrm{C} / \mathrm{A}}(k)$ is spreading coarse acquisition $(\mathrm{C} / \mathrm{A})$ code sequence with a period of 1023 chips, and $f_{\mathrm{L} 1}$ and $\theta_{\mathrm{L} 1}$ are the carrier frequency and the carrier phase of L1.

The beamformer output of GNSS receiver can be written as

$$
y(k)=\mathbf{w}^{H} \mathbf{x}(k)
$$

where $\mathbf{w}$ is the complex beamforming weight vector of the antenna array and $H$ stands for the Hermitian transpose.

The schematic of a linear adaptive antenna array processor on the front end of the GNSS receiver is shown in Figure 1.

\section{Proposed Adaptive Beamforming Scheme for GNSS Receiver}

To attain the aim of low used number sensors for the tight energy control GNSS system and reduce the performance misadjustment of satellite signal acquisition for impulsive noises simultaneously, we detail the proposed constraint sparse maximum correntropy (CSMC) beamforming method for GNSS receiver in this section. In addition, the mean square analysis of CSMC is carried out, and the convergence stability condition of CSMC algorithm is derived using the generalization of approximation methods.

3.1. CSMC Iteration Algorithm. In information learning theory, correntropy is proposed to obtain robustness against outliers. It is a local similarity measure between two arbitrary random variables $\mathrm{A}$ and $\mathrm{B}$ and can be expressed as

$$
V_{\sigma}(A, B)=E\left[k_{\sigma}(A-B)\right]
$$

where $k_{\sigma}$ is a kernel that satisfies the Mercer theory. One of the common choices of $k_{\sigma}$ adopted in this paper is the Gaussian kernel given as

$$
k_{\sigma}(A-B)=\frac{1}{\sigma \sqrt{2 \pi}} \exp \left(-\frac{|A-B|^{2}}{2 \sigma^{2}}\right)
$$

where $\sigma$ is the kernel size. In the field of GNSS array system discussed in this paper, the cost function of the MCC-based algorithm is considered to be the correntropy between the local satellite signal replica and outputs of the beamformer, as given by

$$
J(k)=E\left[\frac{1}{\sigma \sqrt{2 \pi}} \exp \left(-\frac{\left|d(k)-\mathbf{w}^{H} \mathbf{x}(k)\right|^{2}}{2 \sigma^{2}}\right)\right]
$$




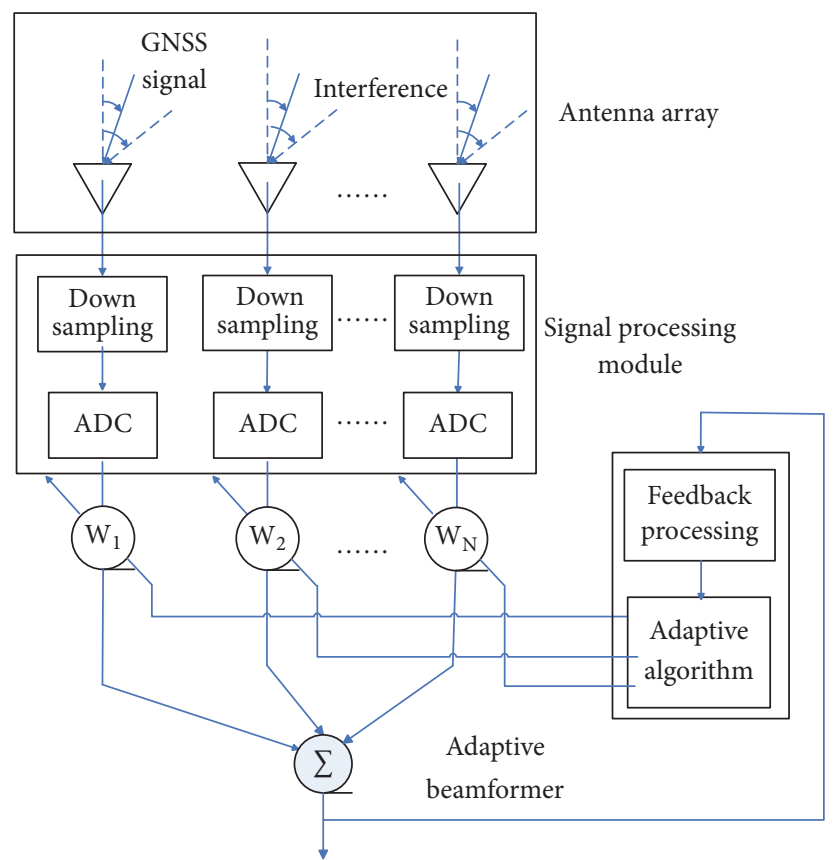

FIGURE 1: The schematic of a linear antenna array processor in GNSS system.

Based on the constrained equations in CLMS [15] and adding a specific L1-norm to the optimization function in (7), we can obtain the following constrained optimization of CSMC:

$$
\begin{array}{ll}
\max _{w} & J(k) \\
\text { s.t. } & \left\{\begin{array}{l}
\mathbf{C}^{H} \mathbf{w}=\mathbf{z} \\
\|\mathbf{w}\|_{1}=t
\end{array}\right.
\end{array}
$$

In order to solve the constrained optimization problem in (8), the Lagrange multiplier and related mathematical skills are used. The object function can be rewritten as

$$
\begin{aligned}
\zeta(\mathbf{w})= & E\left[\frac{1}{\sigma \sqrt{2 \pi}} \exp \left(-\frac{\left|d(k)-\mathbf{w}^{H} \mathbf{x}(k)\right|^{2}}{2 \sigma^{2}}\right)\right] \\
& -\lambda_{1}^{H}\left(\mathbf{C}^{H} \mathbf{w}-\mathbf{z}\right)-\lambda_{2}\left(\|\mathbf{w}\|_{1}-t\right)
\end{aligned}
$$

Using the instantaneous approximation, we can eliminate the expectation operator from (9) as follows:

$$
\begin{aligned}
\zeta(\mathbf{w})= & \frac{1}{\sigma \sqrt{2 \pi}} \exp \left(-\frac{\left|d(k)-\mathbf{w}^{H} \mathbf{x}(k)\right|^{2}}{2 \sigma^{2}}\right) \\
& -\lambda_{1}^{H}\left(\mathbf{C}^{H} \mathbf{w}-\mathbf{z}\right)-\lambda_{2}\left(\|\mathbf{w}\|_{1}-t\right)
\end{aligned}
$$

Calculate the instantaneous gradient of the Lagrange function (10) relative to vector $\mathbf{w}$ concludes to the following relation:

$$
\begin{aligned}
\nabla_{\mathbf{w}} \zeta(\mathbf{w})= & \frac{1}{\sqrt{2 \pi} \sigma^{3}} e(k) \exp \left[-\frac{|e(k)|^{2}}{2 \sigma^{2}}\right] \mathbf{x}(k)-\mathbf{C} \lambda_{1} \\
& -\lambda_{2} \operatorname{sign}[\mathbf{w}]
\end{aligned}
$$

Applying the steepest ascent method, we can obtain the coefficient vector updated at each iteration as

$$
\mathbf{w}(k+1)=\mathbf{w}(k)+\mu \nabla_{\mathbf{w}} \zeta(\mathbf{w})
$$

where $\mu$ is the step size. Inserting the gradient vector from (11) into (12), we can get

$$
\mathbf{w}(k+1)=\mathbf{w}(k)+\mu\left(\mathbf{B}(k)-\mathbf{C} \lambda_{1}-\lambda_{2} \operatorname{sign}[\mathbf{w}(k)]\right)
$$

where $\mathbf{B}(k)=\left(1 / \sqrt{2 \pi} \sigma^{3}\right) e(k) \exp \left[-|e(k)|^{2} / 2 \sigma^{2}\right] \mathbf{x}(k)$.

It is possible to solve for $\lambda_{1}$ premultiplying (13) by $\mathrm{C}^{H}$ and using the constraint relation $\mathbf{C}^{H} \mathbf{w}(k+1)=\mathbf{z}$ in [22], such that

$$
\lambda_{1}=\frac{\mathbf{H w}(k)}{\mu}+\mathbf{H B}(k)-\lambda_{2} \mathbf{H} \operatorname{sign}[\mathbf{w}(k)]-\frac{\mathbf{G z}}{\mu}
$$

where $\mathbf{H}=\left(\mathbf{C}^{H} \mathbf{C}\right)^{-1} \mathbf{C}^{H}$ and $\mathbf{G}=\left(\mathbf{C}^{H} \mathbf{C}\right)^{-1}$.

Due to the constraints in the maximization problem with L1-norm, the approximation $\operatorname{sign}^{H}[\mathbf{w}(k)] \mathbf{w}(k+1) \approx t$ is utilized. Using the definition $t_{k}=\operatorname{sign}^{H}[\mathbf{w}(k)] \mathbf{w}(k)$ and the 
TABLE 1: Summary of the proposed CSMC algorithm.

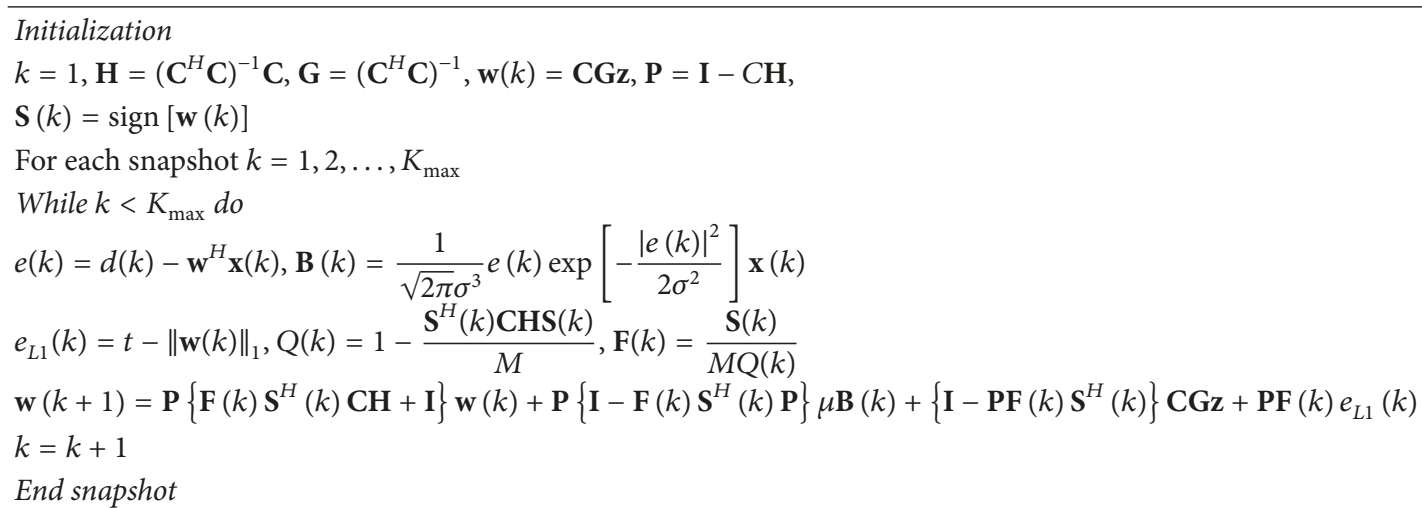

approximation $\operatorname{sign}^{H}[\mathbf{w}(k)] \operatorname{sign}[\mathbf{w}(k)]=M$, it is possible to premultiply (13) by $\operatorname{sign}^{H}[\mathbf{w}(k)]$ and solve for $\lambda_{2}$.

$$
\begin{aligned}
\lambda_{2}= & \frac{\operatorname{sign}^{H}[\mathbf{w}(k)] \mathbf{B}(k)}{M}-\frac{\operatorname{sign}^{H}[\mathbf{w}(k)] \mathbf{C} \lambda_{1}}{M} \\
& -\frac{e_{L 1}(k)}{\mu M}
\end{aligned}
$$

where $e_{L 1}(k)=t-t_{k}$.

It is necessary to solve the system of equations stated by (14) and (15) to obtain the Lagrange multipliers $\lambda_{1}$ and $\lambda_{2}$. Defining $\mathbf{P}=\mathbf{I}-\mathbf{C H}$, the resulting update expression of GNSS array vector presented here can be written as

$$
\begin{aligned}
\mathbf{w}(k+1)= & \mathbf{P} \\
& \left\{\frac{\mathbf{S}(k) \mathbf{S}^{H}(k)}{M Q(k)} \mathbf{C H}+\mathbf{I}\right\} \mathbf{w}(k) \\
& +\mathbf{P}\left\{\mathbf{I}-\frac{\mathbf{S}(k) \mathbf{S}^{H}(k)}{M Q(k)} \mathbf{P}\right\} \mu \mathbf{B}(k) \\
& +\left\{\mathbf{I}-\mathbf{P} \frac{\mathbf{S}(k) \mathbf{S}^{H}(k)}{M Q(k)}\right\} \mathbf{C G z} \\
& +\frac{\mathbf{P S}(k)}{M Q(k)} e_{L 1}(k)
\end{aligned}
$$

with

$$
\begin{aligned}
Q(k) & =1-\frac{\mathbf{S}^{H}(k) \mathbf{C H S}(k)}{M}, \\
\mathbf{S}(k) & =\operatorname{sign}[\mathbf{w}], \\
\mathbf{F}(k) & =\frac{\mathbf{S}(k)}{M Q(k)}
\end{aligned}
$$

Implementation of the CSMC algorithm is summarized in Table 1.

3.2. Mean Square Analysis of CSMC. We perform the mean square analysis of the CSMC algorithm in this subsection; the following commonly used assumptions are given as in the derivations of the convergence behavior of the proposed algorithm: (i) Let $\mathbf{w}_{1 o}$ be the optimal weight vector that takes into account the L1-norm constraint. The weight coefficient deviation vector of the CSMC is defined as $\Delta \mathbf{w}_{1}(k)=\mathbf{w}_{1}(k)-$ $\mathbf{w}_{10}$.

(ii) The desired response produced by the CSMC beamformer of GNSS system can be expressed as $\mathbf{d}_{1}(k)=\mathbf{w}_{1 o}^{H} \mathbf{x}(k)+$ $\mathbf{v}(k) ; \mathbf{v}(k)$ is the uncorrelated additive noise with zero mean and variance $\sigma_{v}^{2}$ of filters and is independent of $\mathbf{x}(k)$.

(iii) The signal $\mathbf{x}(k)$ received by GNSS array is independent and identically distributed (i.i.d.) with zero mean and variance $\sigma_{x}^{2}$. It is approximately independent of the a priori errors and uncorrelated to the noise samples.

In the context of the previously defined CSMC update model (16), let us consider the constraint fact that $\mathbf{C}^{H} \mathbf{w}_{1}(k+$ $1)=\mathbf{C}^{H} \mathbf{w}_{1}(k)=\mathbf{z}$ and simplify the iterative equation of CSMC; we obtain

$$
\mathbf{w}_{1}(k+1)=\mathbf{P}\left[\mathbf{w}_{1}(k)+\mu \mathbf{M}(k) \mathbf{B}(k)\right]+\mathbf{F}+\mathbf{F}_{L 1}(k)
$$

with

$$
\begin{aligned}
\mathbf{M}(k) & =\mathbf{I}-\frac{\mathbf{P S}(k) \mathbf{S}^{H}(k)}{\|\mathbf{P S}(k)\|^{2}}, \\
\mathbf{F} & =\mathbf{C G z}, \\
\mathbf{F}_{L 1}(k) & =\left(\frac{\mathbf{P S}(k)}{\|\mathbf{P S}(k)\|^{2}}\right) e_{L 1}(k)
\end{aligned}
$$

With the assumption $\Delta \mathbf{w}_{1}(k)=\mathbf{w}_{1}(k)-\mathbf{w}_{1 o}$ and the definitions $\mathbf{V}(k)=\mathbf{P S}(k) /\|\mathbf{P S}(k)\|^{2}$ and $\mathbf{M}_{o}(k)=$ $\mathbf{P S}(k) \mathbf{S}^{H}(k) /\|\mathbf{P S}(k)\|^{2}$, the constraint vector $\mathbf{F}$ and the auxiliary vector $\mathbf{F}_{L 1}$ can be, respectively, stated as

$$
\mathbf{F}=(\mathbf{I}-\mathbf{P}) \mathbf{w}_{1 o}
$$

and

$$
\begin{aligned}
\mathbf{F}_{L 1} & =\left(t-\mathbf{S}^{H}(k) \mathbf{w}_{1}(k)\right) \mathbf{V}(k) \\
& =\left(t-\mathbf{S}^{H}(k) \Delta \mathbf{w}_{1}(k)-\mathbf{S}^{H}(k) \mathbf{w}_{1 o}\right) \mathbf{V}(k) \\
& =t \mathbf{V}(k)-\mathbf{P} \mathbf{M}_{o}(k)\left(\Delta \mathbf{w}_{1}(k)+\mathbf{w}_{1 o}\right)
\end{aligned}
$$


Using the formulation from (20) and (21), the resulting update equation of weight deviation vector can be computed as

$$
\begin{aligned}
\Delta \mathbf{w}_{1}(k+1)= & \mathbf{P}\left(\mathbf{I}-\mathbf{M}_{o}(k)\right) \Delta \mathbf{w}_{1}(k)-\mathbf{P} \mathbf{M}_{o}(k) \mathbf{w}_{1 o} \\
& +\mu \mathbf{P}\left(\mathbf{I}-\mathbf{M}_{o}(k)\right) \mathbf{B}(k)+t \mathbf{V}(k)
\end{aligned}
$$

Substituting the equations $\mathbf{d}_{1}(k)=\mathbf{w}_{1 o}^{H} \mathbf{x}(k)+\mathbf{v}(k)$ and $\mathbf{e}(k)=$ $\mathbf{d}_{1}(k)-\mathbf{w}_{1}^{H}(k) \mathbf{x}(k)$ in (22), we obtain

$$
\begin{aligned}
\Delta \mathbf{w}_{1}(k+1)= & \mathbf{A}(k) \mathbf{H}(k) \Delta \mathbf{w}_{1}(k)-\mathbf{P} \mathbf{M}_{o}(k) \mathbf{w}_{1 o} \\
& +m u \mathbf{v}(k) f(e(k)) \mathbf{A}(k) \mathbf{x}(k) \\
& +t \mathbf{V}(k)
\end{aligned}
$$

with

$$
\begin{aligned}
\mathbf{A}(k) & =\mathbf{P}\left(\mathbf{I}-\mathbf{M}_{o}(k)\right), \\
\mathbf{H}(k) & =\mathbf{I}-m u f(e(k)) \mathbf{x}(k) \mathbf{x}^{H}(k), \\
f(e(k)) & =\exp \left[-\frac{|e(k)|^{2}}{2 \sigma^{2}}\right], \\
m u & =\frac{\mu}{\sqrt{2 \pi} \sigma^{3}}
\end{aligned}
$$

The error nonlinearity $f(e(k))$ is independent of the input signal $\mathbf{x}(k)$ and weight $\mathbf{w}_{1}(k)$.

Taking the expected value of (23) and using the independence assumption [23], we get

$$
\begin{aligned}
E[ & \left.\Delta \mathbf{w}_{1}(k+1)\right] \\
= & \mathbf{A}(k)\left\{1-m u E[f(e(k))] \sigma_{x}^{2}\right\} E\left[\Delta \mathbf{w}_{1}(k)\right] \\
& \quad-E\left[\mathbf{P M}_{o}(k) \mathbf{w}_{1 o}\right]+t E[\mathbf{V}(k)]
\end{aligned}
$$

To perform the mean square convergence of the CSMC algorithm, we define the autocovariance of $\Delta \mathbf{w}_{1}(k)$ as

$$
\mathbf{R}(k)=E\left[\mathbf{Z}(k) \mathbf{Z}^{T}(k)\right]
$$

where $\mathbf{Z}(k)$ denotes the zero-mean misalignment vector as

$$
\mathbf{Z}(k)=\Delta \mathbf{w}_{1}(k)-E\left[\Delta \mathbf{w}_{1}(k)\right]
$$

Now, subtracting (25) from (23) and substituting (27) give

$$
\begin{aligned}
\mathbf{Z}(k+1)= & \mathbf{A}(k) \mathbf{H}(k) \mathbf{Z}(k)+\mathbf{D}(k)+\mathbf{W}(k)+\mathbf{L}(k) \\
& +\mathbf{H}_{1}(k) E\left[\Delta \mathbf{w}_{1}(k)\right]
\end{aligned}
$$

where

$$
\begin{aligned}
\mathbf{H}_{1}(k) & =\mathbf{A}(k)\left\{\mathbf{H}(k)+m u E[f(e(k))] \sigma_{x}^{2}-1\right\} \\
\mathbf{D}(k) & =E\left[\mathbf{P M}_{o}(k) \mathbf{w}_{1 o}\right]-\mathbf{P M}_{o}(k) \mathbf{w}_{1 o} \\
\mathbf{W}(k) & =\operatorname{muv}(k) f(e(k)) \mathbf{A}(k) \mathbf{x}(k) \\
\mathbf{L}(k) & =t \mathbf{V}(k)-E[t \mathbf{V}(k)]
\end{aligned}
$$

It is straightforward to verify that $\mathbf{D}(k), \mathbf{L}(k)$, and $\mathbf{H}_{1}(k)$ are of zero mean. Besides, with the independence assumption, i.e., $\Delta \mathbf{w}_{1}(k), \mathbf{x}(k)$, and $v(k)$ are mutually independent [24], and substituting (28) into (26), we can derive the resulting $\mathbf{R}(k+1)$ after some tedious calculations

$$
\begin{aligned}
& \mathbf{R}(k+1)=E\left[\begin{array}{c}
\left(\begin{array}{c}
\mathbf{A}(k) \mathbf{H}(k) \mathbf{Z}(k)+\mathbf{D}(k)+\mathbf{W}(k) \\
+\mathbf{L}(k)+\mathbf{H}_{1}(k) E\left[\Delta \mathbf{w}_{1}(k)\right]
\end{array}\right) \\
\times\left(\begin{array}{c}
\mathbf{A}(k) \mathbf{H}(k) \mathbf{Z}(k)+\mathbf{D}(k)+\mathbf{W}(k) \\
+\mathbf{L}(k)+\mathbf{H}_{1}(k) E\left[\Delta \mathbf{w}_{1}(k)\right]
\end{array}\right)^{T}
\end{array}\right] \\
& =\left[\begin{array}{c}
E\left[\mathbf{A}(k) \mathbf{H}(k) \mathbf{Z}(k) \mathbf{Z}^{T}(k) \mathbf{H}^{T}(k) \mathbf{A}^{T}(k)\right]+E\left[\mathbf{L}(k) \mathbf{L}^{T}(k)\right] \\
+E\left[\mathbf{A}(k) \mathbf{H}(k) \mathbf{Z}(k) \mathbf{D}^{T}(k)\right]+E\left[\mathbf{A}(k) \mathbf{H}(k) \mathbf{Z}(k) \mathbf{L}^{T}(k)\right] \\
+E\left[\mathbf{D}(k) \mathbf{Z}^{T}(k) \mathbf{H}^{T}(k) \mathbf{A}^{T}(k)\right]+E\left[\mathbf{D}(k) \mathbf{D}^{T}(k)\right] \\
+E\left[\mathbf{W}(k) \mathbf{W}^{T}(k)\right]+E\left[\mathbf{L}(k) \mathbf{Z}^{T}(k) \mathbf{H}^{T}(k) \mathbf{A}^{T}(k)\right] \\
+E\left[\mathbf{H}_{1}(k) E\left[\Delta \mathbf{w}_{1}(k)\right] E^{T}\left[\Delta \mathbf{w}_{1}(k)\right] \mathbf{H}_{1}^{T}(k)\right]
\end{array}\right]
\end{aligned}
$$


To evaluate (33), using the fact that the fourth-order moment of a Gaussian variable is three times the variance square [23], and by the independence assumption and symmetric behavior of the covariance matrix [24], we obtain

$$
\begin{gathered}
E\left[\mathbf{A}(k) \mathbf{H}(k) \mathbf{Z}(k) \mathbf{Z}^{T}(k) \mathbf{H}^{T}(k) \mathbf{A}^{T}(k)\right]=\mathbf{A}(k) \\
\cdot\left\{\begin{array}{c}
\left\{\begin{array}{c}
1-2 m u E[f(e(k))] \sigma_{x}^{2} \\
+2(m u)^{2} \sigma_{x}^{4} E\left[f^{2}(e(k))\right]
\end{array}\right\} \mathbf{R}(k) \\
+(m u)^{2} \sigma_{x}^{4} E\left[f^{2}(\mathrm{e}(k))\right] \operatorname{tr}(\mathbf{R}(k)) \mathbf{I}_{M}
\end{array}\right\} \mathbf{A}^{T}(k)
\end{gathered}
$$

where $\operatorname{tr}(\bullet)$ denotes the trace of a matrix. Also, with (23)-(28), we can get the other terms in (33)

$$
\begin{aligned}
E & {\left[\mathbf{A}(k) \mathbf{H}(k) \mathbf{Z}(k) \mathbf{D}^{T}(k)\right] } \\
& =E^{T}\left[\mathbf{D}(k) \mathbf{Z}^{T}(k) \mathbf{H}^{T}(k) \mathbf{A}^{T}(k)\right] \\
& =\left\{1-m u E\left[f^{2}(e(k))\right] \sigma_{x}^{2}\right\} E\left[\mathbf{A}(k) \mathbf{w}(k) \mathbf{D}^{T}(k)\right] \\
E & {\left[\mathbf{A}(k) \mathbf{H}(k) \mathbf{Z}(k) \mathbf{L}^{T}(k)\right] } \\
& =E^{T}\left[\mathbf{L}(k) \mathbf{Z}^{T}(k) \mathbf{H}^{T}(k) \mathbf{A}^{T}(k)\right] \\
& =\left\{1-m u E\left[f^{2}(e(k))\right] \sigma_{x}^{2}\right\} E\left[\mathbf{A}(k) \mathbf{w}(k) \mathbf{L}^{T}(k)\right]
\end{aligned}
$$

$$
\begin{aligned}
\operatorname{tr}(\mathbf{R}(k+1))= & \operatorname{tr}\left(\mathbf{A}(k)\left\{\begin{array}{c}
\left\{\begin{array}{c}
1-2 m u E[f(e(k))] \sigma_{x}^{2} \\
+2(m u)^{2} \sigma_{x}^{4} E\left[f^{2}(e(k))\right]
\end{array}\right\} \mathbf{R}(k) \\
+(m u)^{2} \sigma_{x}^{4} E\left[f^{2}(e(k))\right] \operatorname{tr}(\mathbf{R}(k)) \mathbf{I}_{M}
\end{array}\right\} \mathbf{A}^{T}(k)\right) \\
& +2\left\{1-m u E\left[f^{2}(e(k))\right] \sigma_{x}^{2}\right\} E\left[\mathbf{A}(k) \mathbf{w}(k) \mathbf{D}^{T}(k)\right] \\
& +2\left\{1-m u E\left[f^{2}(e(k))\right] \sigma_{x}^{2}\right\} E\left[\mathbf{A}(k) \mathbf{w}(k) \mathbf{L}^{T}(k)\right]+E\left[\mathbf{D}(k) \mathbf{D}^{T}(k)\right]+E\left[\mathbf{L}(k) \mathbf{L}^{T}(k)\right] \\
& +(m u)^{2} \sigma_{v}^{2} \sigma_{x}^{2} E\left[f^{2}(e(k))\right] \mathbf{A}(k) \mathbf{A}^{T}(k) \\
& +(m u)^{2} E\left[f^{2}(e(k))\right] \sigma_{x}^{4} t r\left(\mathbf{A}(k)\left[\begin{array}{c}
\left.\operatorname{tr}\left(\boldsymbol{\delta}(k) \boldsymbol{\delta}^{T}(k)\right) \mathbf{I}_{M}\right] \\
+\boldsymbol{\delta}(k) \boldsymbol{\delta}^{T}(k)
\end{array}\right] \mathbf{A}^{T}(k)\right)
\end{aligned}
$$

Using the properties of the trace of matrix, we can expand the first term of (40) through the inequality of traces products [25]; we have

$$
\begin{aligned}
& \operatorname{tr}\left(\mathbf{A}(k)\left\{\begin{array}{c}
\left\{\begin{array}{c}
1-2 m u E[f(e(k))] \sigma_{x}^{2} \\
+2(m u)^{2} \sigma_{x}^{4} E\left[f^{2}(e(k))\right]
\end{array}\right\} \mathbf{R}(k) \\
+(m u)^{2} \sigma_{x}^{4} E\left[f^{2}(e(k))\right] \operatorname{tr}(\mathbf{R}(k)) \mathbf{I}_{M}
\end{array}\right\} \mathbf{A}^{T}(k)\right) \\
& \leq \operatorname{tr}\left(\begin{array}{c}
\left\{\begin{array}{c}
1-2 m u E[f(e(k))] \sigma_{x}^{2} \\
+2(m u)^{2} \sigma_{x}^{4} E\left[f^{2}(e(k))\right]
\end{array}\right\} \mathbf{R}(k) \\
+(m u)^{2} \sigma_{x}^{4} E\left[f^{2}(e(k))\right] \operatorname{tr}(\mathbf{R}(k)) \mathbf{I}_{M}
\end{array}\right) \operatorname{tr}\left(\mathbf{A}(k) \mathbf{A}^{T}(k)\right) \\
& =\left\{\begin{array}{c}
1-2 m u E[f(e(k))] \sigma_{x}^{2} \\
+(M+2)(m u)^{2} \sigma_{x}^{4} E\left[f^{2}(e(k))\right]
\end{array}\right\}\left(\begin{array}{c}
\operatorname{tr}\left(\mathbf{P P}^{T}\right) \\
-2 \operatorname{tr}\left(\mathbf{P M}_{o}^{T}(k) \mathbf{P}^{T}\right) \\
-\operatorname{tr}\left(\mathbf{P M}_{o}(k) \mathbf{M}_{o}^{T}(k) \mathbf{P}^{T}\right)
\end{array}\right) \operatorname{tr}(\mathbf{R}(k))
\end{aligned}
$$



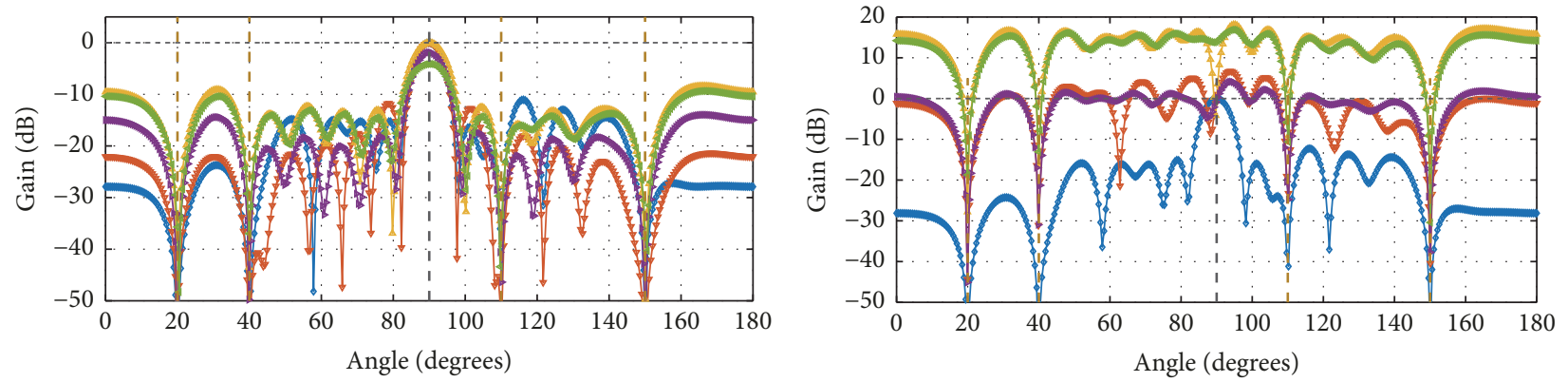

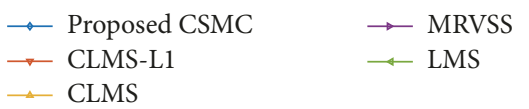

(a)

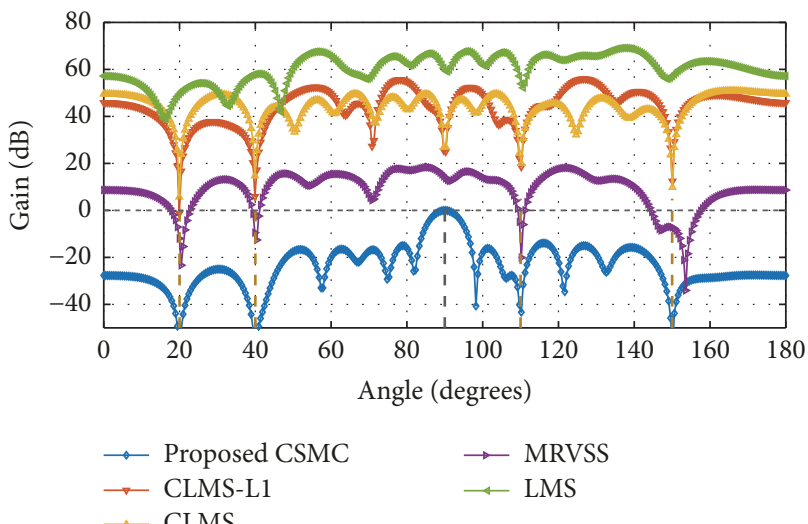

(c)

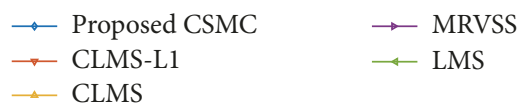

(b)

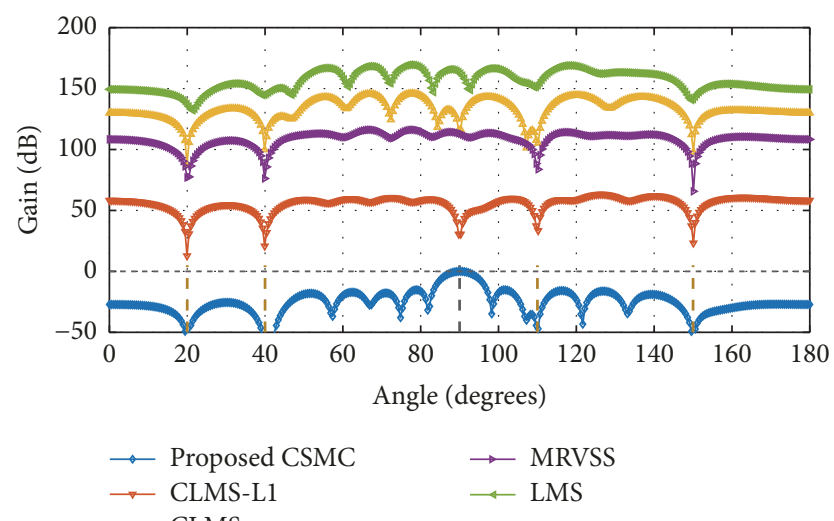

(d)

FiguRE 2: Beampatterns of the proposed and the other existing beamformers with different $\alpha$ : (a) $\alpha=1.65$; (b) $\alpha=1.5$; (c) $\alpha=1.35$; (d) $\alpha=1.2$.

From (41), it can be obviously seen that $\boldsymbol{\delta}(k), E[\mathbf{w}(k)]$, $\mathbf{L}(k), \mathbf{D}(k), E[\mathbf{A}(k)]$ are all bounded terms [23, 24], and thus we can state that, in order to ensure convergence, it is necessary to maintain the trace of the second-order moment within the interval $[0,1]$; therefore, the adaptive beamformer is stable if and only if

$$
\left|\left\{\begin{array}{c}
1-2 m u E[f(e(k))] \sigma_{x}^{2} \\
+(M+2)(m u)^{2} \sigma_{x}^{4} E\left[f^{2}(e(k))\right]
\end{array}\right\}\left(\begin{array}{c}
\operatorname{tr}\left(\mathbf{P P}^{T}\right)-2 \operatorname{tr}\left(\mathbf{P} \mathbf{M}_{o}^{T}(k) \mathbf{P}^{T}\right) \\
-\operatorname{tr}\left(\mathbf{P M}_{o}(k) \mathbf{M}_{o}^{T}(k) \mathbf{P}^{T}\right)
\end{array}\right)\right| \leq 1
$$

To further evaluate the mean square analysis, we apply the principle of statistical independence to (42) and expand the trace of matrix through an inequality of trace products for positive semidefinite matrices of the same order; we obtain

$$
\begin{aligned}
& \operatorname{tr}\left(\mathbf{P} \mathbf{P}^{T}\right)-2 \operatorname{tr}\left(\mathbf{P M}_{o}^{T}(k) \mathbf{P}^{T}\right) \\
& \quad-\operatorname{tr}\left(\mathbf{P M}_{o}(k) \mathbf{M}_{o}^{T}(k) \mathbf{P}^{T}\right)=M+L^{2}-2 L+3 \\
& \quad+\operatorname{tr}\left(\mathbf{C H M}_{o}(k)(\mathbf{C H})^{T}\right) \\
& \quad+\operatorname{tr}\left(\mathbf{C H M}_{o}(k) \mathbf{M}_{o}^{T}(k)(\mathbf{C H})^{T}\right)
\end{aligned}
$$

$$
\begin{aligned}
& -2 \operatorname{tr}\left(\mathbf{M}_{o}(k) \mathbf{M}_{o}^{T}(k)(\mathbf{C H})^{T}\right) \geq M+L^{2}-2 L+3 \\
& +2 \operatorname{tr}\left(\mathbf{C H M}_{o}(k)(\mathbf{C H})^{T}\right) \\
& +\operatorname{tr}\left(\mathbf{C H M}_{o}(k) \mathbf{M}_{o}^{T}(k)(\mathbf{C H})^{T}\right) \\
& -2 \operatorname{tr}\left(\mathbf{M}_{o}(k)\right) \operatorname{tr}\left(\mathbf{M}_{o}^{T}(k)\right) \operatorname{tr}\left((\mathbf{C H})^{T}\right)=M \\
& +(L-2)^{2}-1+2 \operatorname{tr}\left(\mathbf{C H M}_{o}(k)(\mathbf{C H})^{T}\right) \\
& +\operatorname{tr}\left(\mathbf{C H M}_{o}(k) \mathbf{M}_{o}^{T}(k)(\mathbf{C H})^{T}\right)
\end{aligned}
$$




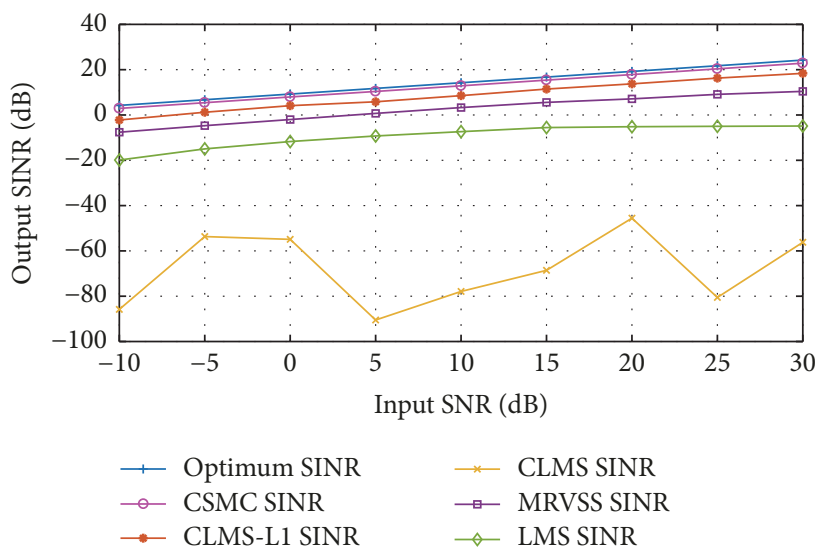

(a)

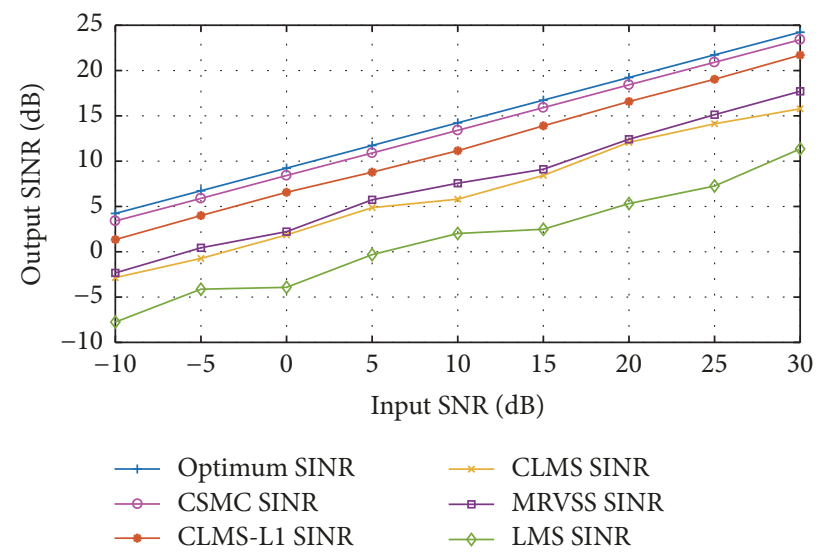

(b)

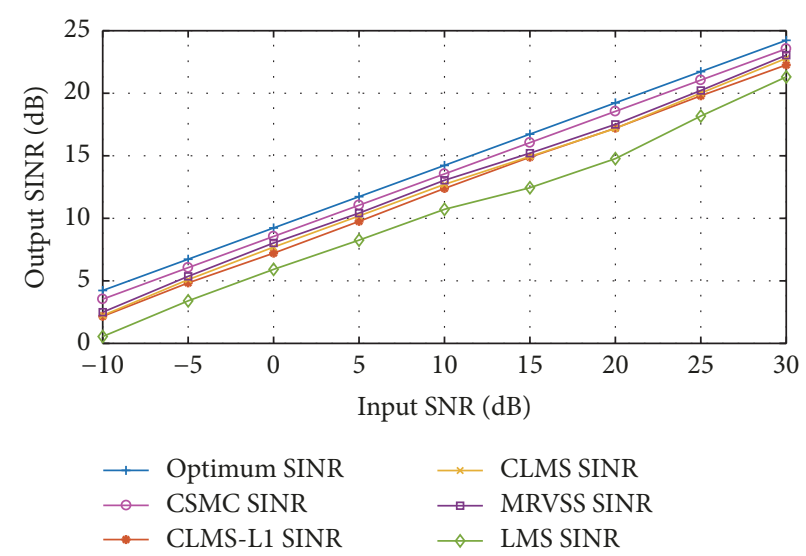

(c)

FIGURE 3: SINR performance against SNR with different $\alpha$ : (a) $\alpha=1.05$; (b) $\alpha=1.25$; (c) $\alpha=1.45$.

Using the properties that the trace of vector is greater than or equal to one and the number of elements in array is more than one by far, we have

$$
\begin{aligned}
& \operatorname{tr}\left(\mathbf{P} \mathbf{P}^{T}\right)-2 \operatorname{tr}\left(\mathbf{P} \mathbf{M}_{o}^{T}(k) \mathbf{P}^{T}\right) \\
& \quad-\operatorname{tr}\left(\mathbf{P} \mathbf{M}_{o}(k) \mathbf{M}_{o}^{T}(k) \mathbf{P}^{T}\right)>>1
\end{aligned}
$$

Therefore, using (44), inequality (42) reduces to

$$
\begin{aligned}
\mid 1 & -2 m u E[f(e(k))] \sigma_{x}^{2} \\
& +(M+2)(m u)^{2} \sigma_{x}^{4} E\left[f^{2}(e(k))\right] \mid<1
\end{aligned}
$$

Hence, combining (45) and the relation between $m u$ and step size $\mu$, we can obtain the range of $\mu$ to meet the mean square stability

$$
0<\mu<\frac{2}{(M+2) E[f(e(k))] \sigma_{x}^{2} / \sqrt{2 \pi} \sigma^{3}}
$$

The deriving result in (46) satisfies the theoretical requirements for convergence step size of adaptive algorithm [26] and confirms the validity of proposed CSMC beamforming method.

\section{Simulation Results}

In this section, several groups of simulation experiments are conducted using Matlab R2015b to verify the proposed CSMC beamforming algorithm for GNSS receivers; a uniform linear array of 15 omnidirectional antenna elements with the interelement spacing of half wavelength is considered in the simulation; the desired satellite signal is the form of $\mathrm{C} / \mathrm{A}$ code with $90^{\circ}$ for incident angle. The carrier frequencies of GNSS signal are set at $1575.42 \mathrm{MHz}$ of the L1 center frequency. The sampling frequency is $5 \mathrm{MHz}$ and the acquisition time is set to $20 \mathrm{~ms}(\mathrm{~K}=100000)$. The code phase delay and the Doppler frequency shift of the received GNSS signal are set to 300 sampling points and $-5 \mathrm{kHz}$, respectively. The step size of Doppler frequency search is $500 \mathrm{~Hz}$. We consider that four single frequency interferences with 45 interference-to-noise ratio (INR) are located at 20,40,110, and 150, respectively.

Additive noise in array elements is modeled as Gaussian noise with zero mean and unit variance. The impulsive noises applied in this paper consider to be a four-parameter family of alpha noises and can be denoted as $S\left(\alpha, \beta_{\alpha}, \gamma, \delta_{\alpha}\right), \alpha \in(0,2]$ is the characteristic exponent, $\beta_{\alpha} \in[-1,1]$ is the skewness, and the last two parameters $\gamma>0, \delta_{\alpha} \in R$ denote the scale and the location of the model. 


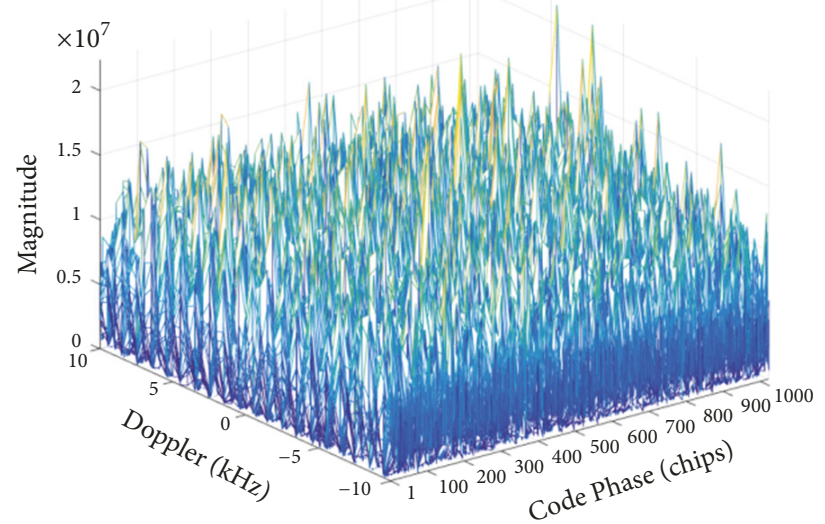

(a)

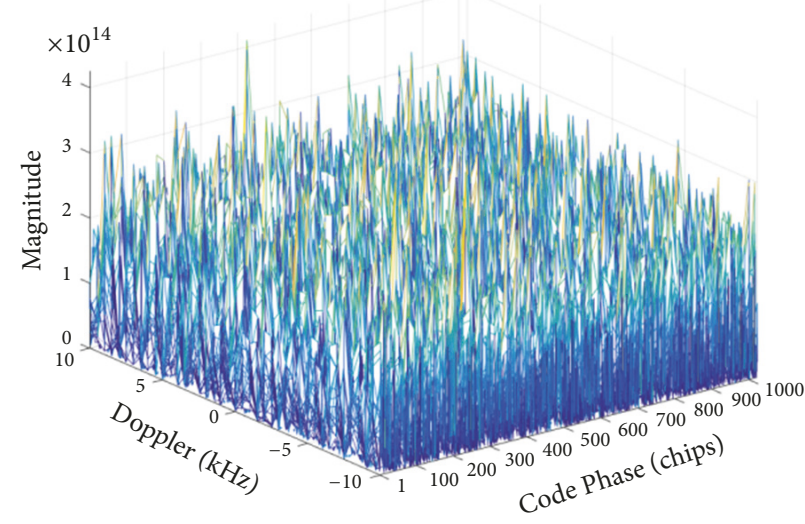

(c)

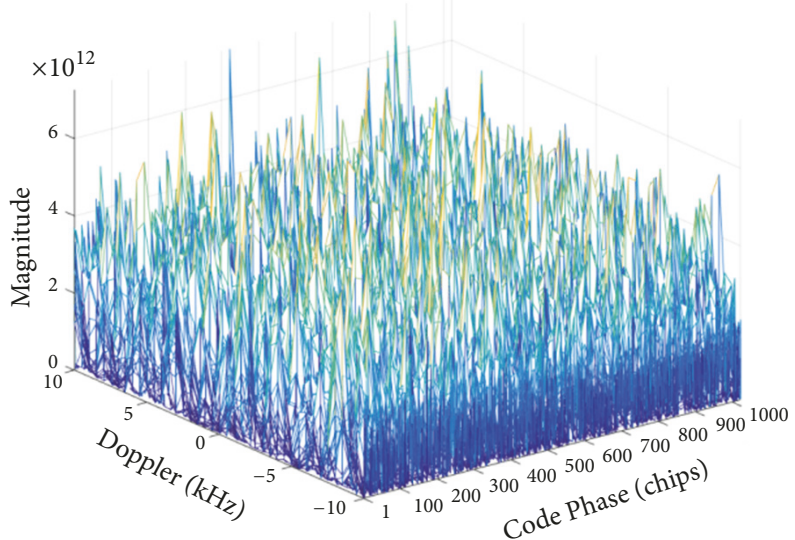

(b)

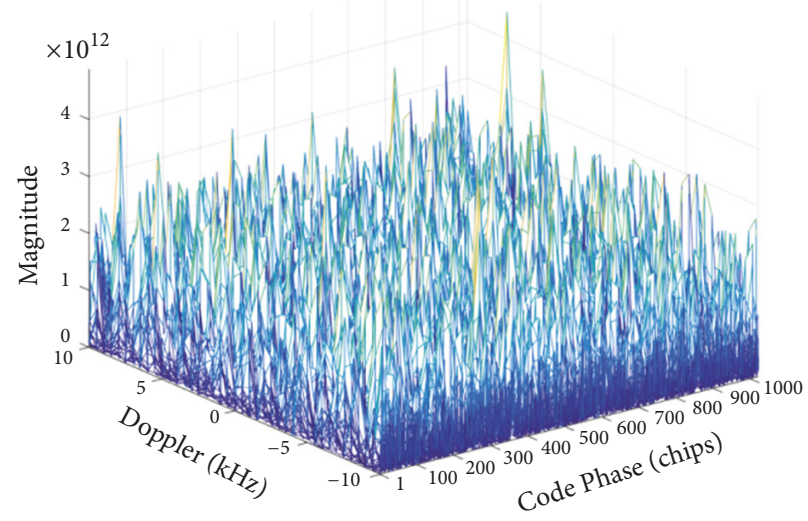

(d)

FIGURE 4: Acquisition results in the impulsive noises with traditional adaptive beamformers in GNSS receiver: (a) CLMS-L1; (b) MRVSS; (c) CLMS; (d) LMS.

The performance for GNSS system with the proposed method is evaluated from the following aspects: the beampattern performance of the array antenna for satellite system receiver, the steady state output SINR of the proposed CSMC beamformer, the acquisition results of the code phase delay and the Doppler frequency shift in time-frequency domain, and the probability of detection for the satellite signal. The sparsity performance of GNSS array induced by the proposed algorithm is also evaluated. All simulation results in the figures are obtained by averaging 200 trials. The proposed methods are compared with the four conventional beamforming methods. The Linear Constrained Minimum Variance (LCMV) solution is used as a closed-form expression to the uniformly optimum weight vector $\mathbf{w}_{\text {opt }}$. All the other parameters used in the simulations are summarized in Table 2.

4.1. Beampattern Performance Comparison. In the first example, we compare the power patterns formed by the different types of beamformers using ULA. The signal-to-noise ratio (SNR) of GNSS signal is $-5 \mathrm{~dB}$. All the beamformers are designed to operate for the impulsive noise scenario. Figures
TABLE 2: Parameter values for MSE.

\begin{tabular}{lc}
\hline Parameter & ULA \\
\hline Kernel Size $\sigma$ & 3 \\
\hline Intermediate Frequency & $1.25 \mathrm{MHz}$ \\
\hline Skewness $\beta_{\alpha}$ & 0 \\
\hline Parameter $\delta_{\alpha}$ & 0 \\
\hline Parameter $\gamma$ & 1 \\
\hline
\end{tabular}

2(a)-2(d) show the beampatterns in presence of the impulsive noise with varied characteristic exponent representing different impulsive levels of noises. As can be seen, the proposed algorithms have suppressed the jammers in all cases while maintaining the beampattern gain in the direction of the desired signal. The other algorithms are able to achieve the similar beamforming performance for lowly impulsive noises, as impulsive degree of the noise interference increase, the four compared beamforming methods suffer from performance degradation of corresponding beampatterns, and the proposed algorithm has evident advantages over these algorithms, especially in the most severely impulsive noise 


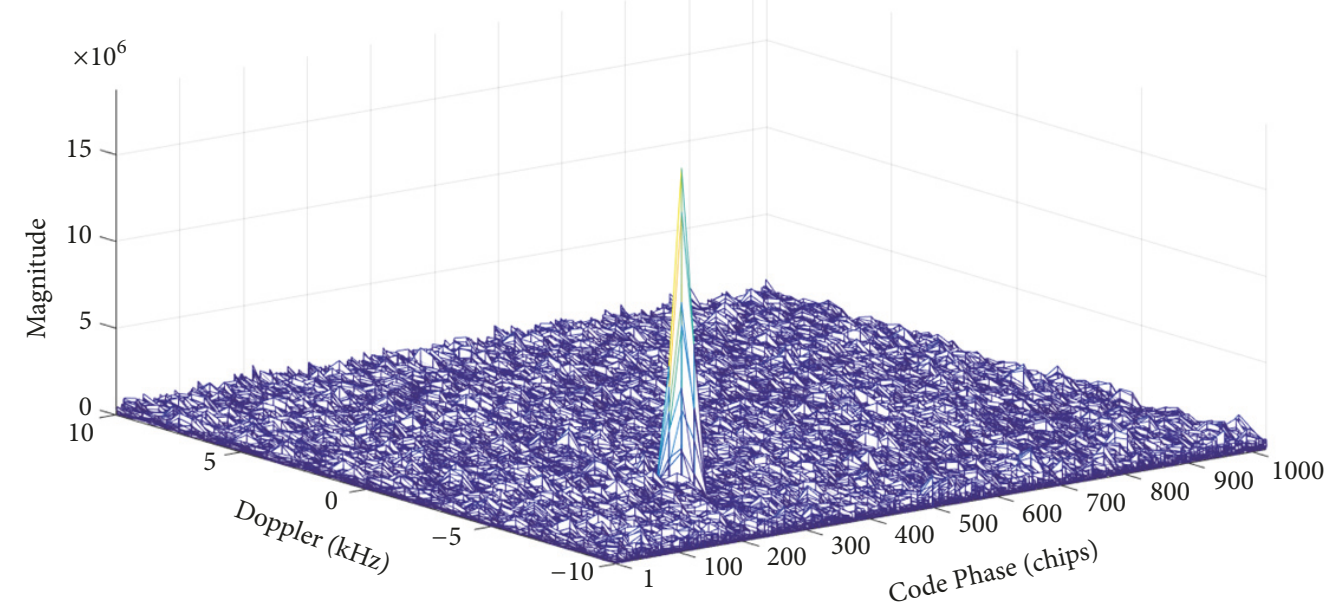

(a)

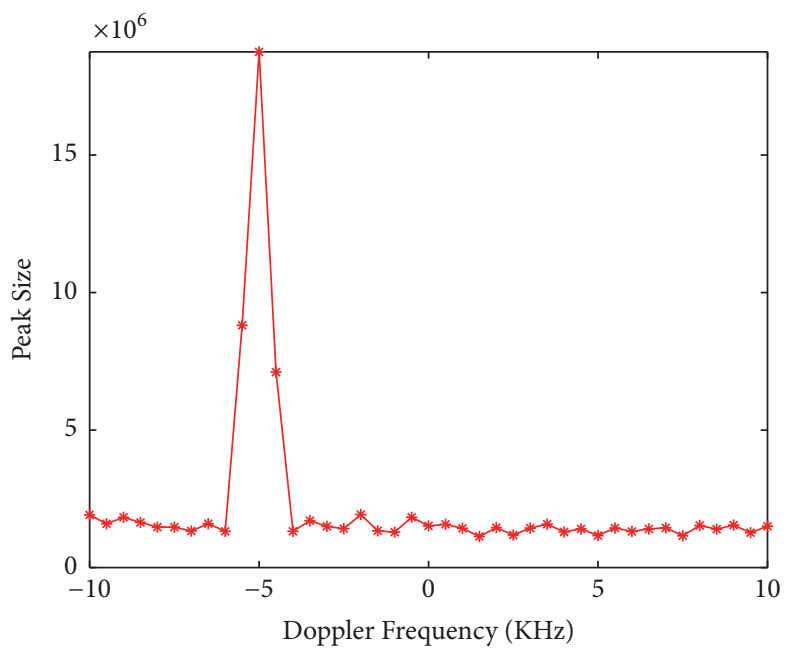

(b)

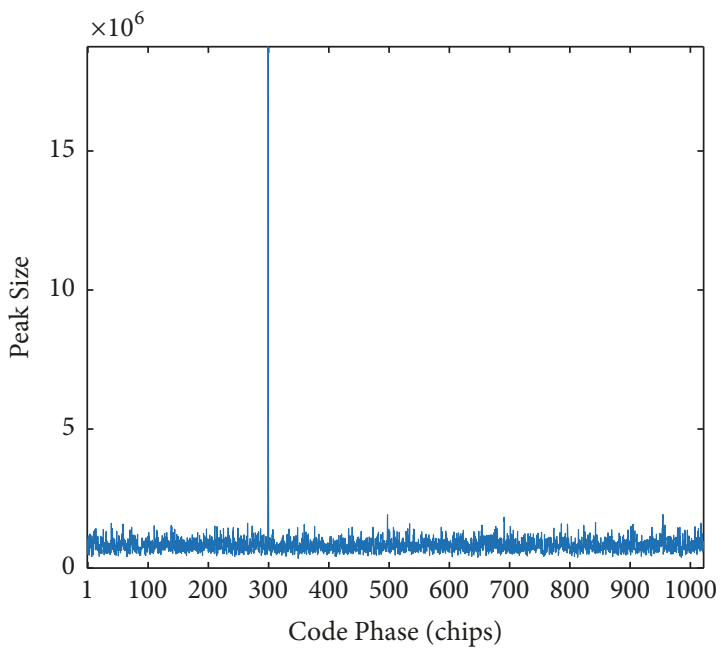

(c)

FIGURE 5: Acquisition results in the impulsive noises with the CSMC beamformer in GNSS receiver: (a) three-dimension domain; (b) the code phase; (c) the Doppler frequency.

situation. Hence, the proposed CSMC achieved improved robustness against impulsive noises than their competitors.

4.2. Output SINR Performance. For the simulation presented in this example, the output SINR performance for the CSMC beamformer is investigated in terms of SNR. We compare the proposed technique to the four aforementioned adaptive beamforming methods. The simulation scenario and parameters setup are the same as used in Section 4.1. When the SNR varies from -10 to $30 \mathrm{~dB}$, the resulting curves are shown in Figure 3. It can be seen from the figures that the SINR of all algorithms increase with higher SNR, but the increasing rates of the comparisons in the simulation show fluctuations gradually as $\alpha$ decreasing, which is due to the heavier disturbance of impulsive noises. The proposed beamforming algorithm leads to the improved SINR performance, especially in the situation of $\alpha=1.05$, which means the most impulsive process of the noises. This fact is due to the MCC criterion which is much more robust against impulsive outliers than MSE estimation. Absolutely, the proposed methods outperform the other algorithms in all the cases and are close to the optimum SINR.

4.3. Adaptive Beamforming for GNSS Signal Acquisition. In this part, we investigate the GNSS signal acquisition performance using regular searching method [27] with the proposed beamforming method; four methods mentioned above are compared in terms of the code phase delay and the Doppler frequency shift acquisition. The input signalto-noise ratio (SNR) is set as $8 \mathrm{~dB}$. Figures $4(\mathrm{a})-4(\mathrm{~d})$ show the three-dimension search results for the GNSS signal in impulsive noises with $\alpha=1.25$ that was acquired with traditional beamformers. We can observe from the figures that hardly any dominant peak could be observed at the correct code phase-Doppler frequency shift in combination with the comparison beamforming algorithms. Peaks 

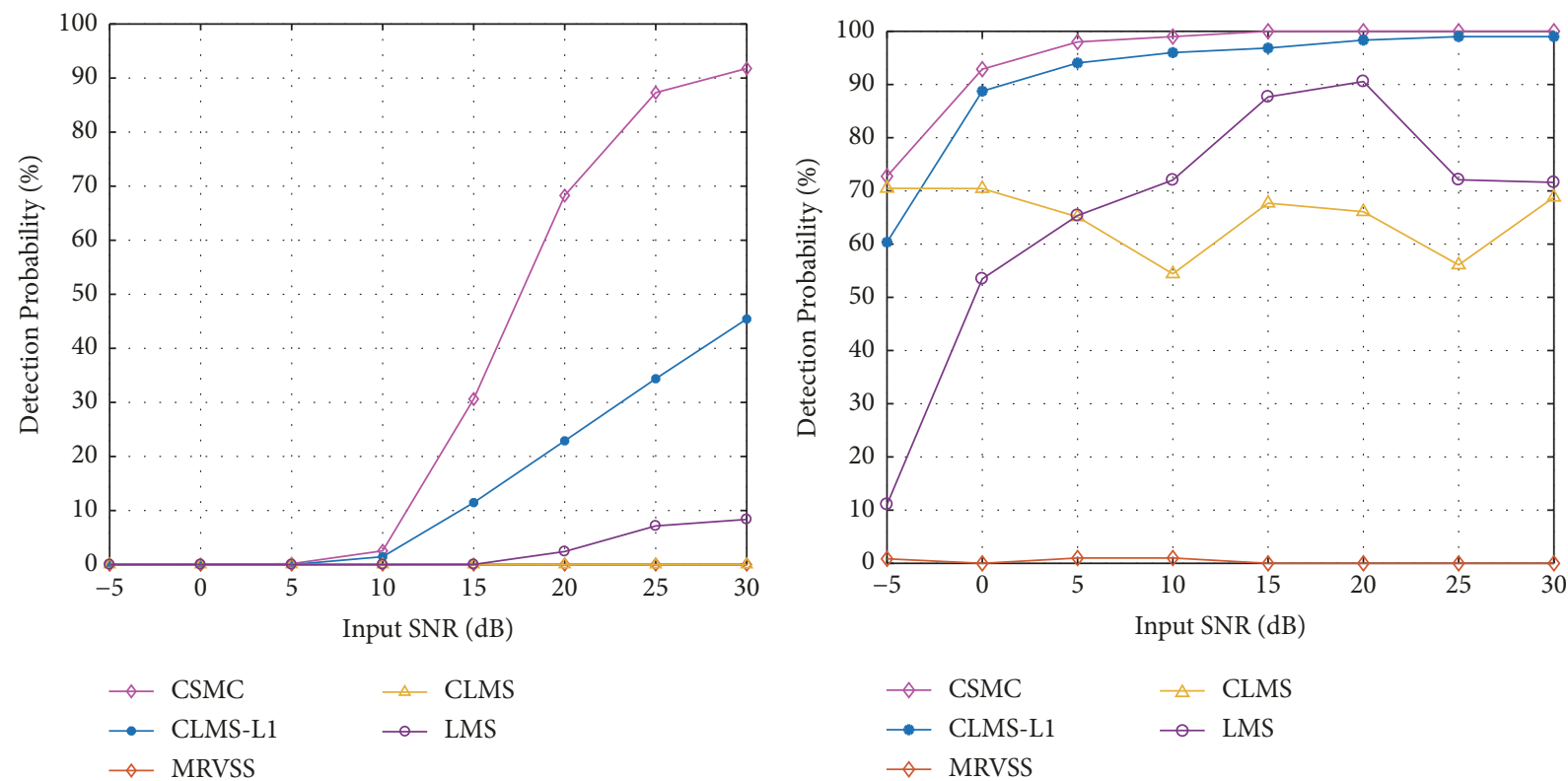

(a)

(b)

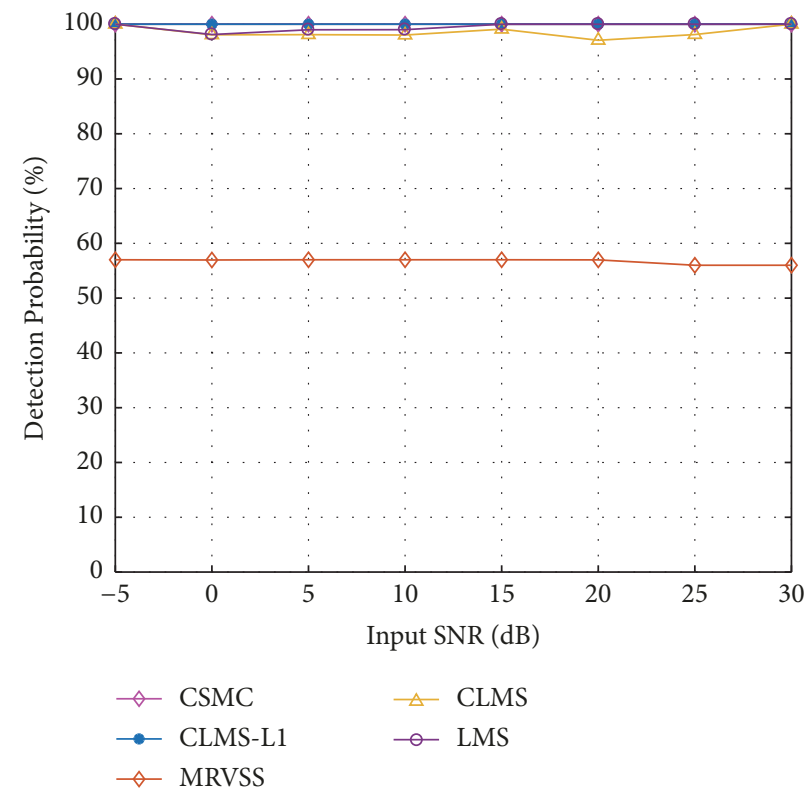

(c)

FIGURE 6: Acquisition performance in terms of detection probability in presence of the alpha noises of different impulsive level: (a) $\alpha=1.05$; (b) $\alpha=1.45$; (c) $\alpha=1.85$.

of indistinct and irregular magnitude exist due to noises and interferences disturbance, which indicates that CLMSL1, MRVSS CLMS, and LMS are susceptible to impulsive noises and the acquisition of the GNSS signal with these techniques fails. The correlation peak with the proposed CSMC beamforming method is depicted in Figure 5(a), as evidenced by the figure, the impact of impulsive noises is drastically reduced, and a significant peak is present in timefrequency domain. The sharp peak occurs at correct code phase $=300$ sampling points and Doppler frequency shift $=-$ $5 \mathrm{KHz}$ which is shown in Figures 5(b) and 5(c). The success of the acquisition with CSMC is due to the novel beamforming criterion used in the GNSS receiver.

4.4. Detection Probability for Acquisition Performance. In this subsection, the acquisition performance with CSMC beamformer is assessed in terms of the detection probability. To check the robustness validity of our approach, we consider three sets of parameters with $\alpha=1.05, \alpha=1.45$, and $\alpha=1.85$ representing the alpha noises of different impulsive level. The acquisition test statistic and detection metric used in this paper is based on the test detector in [28]. 


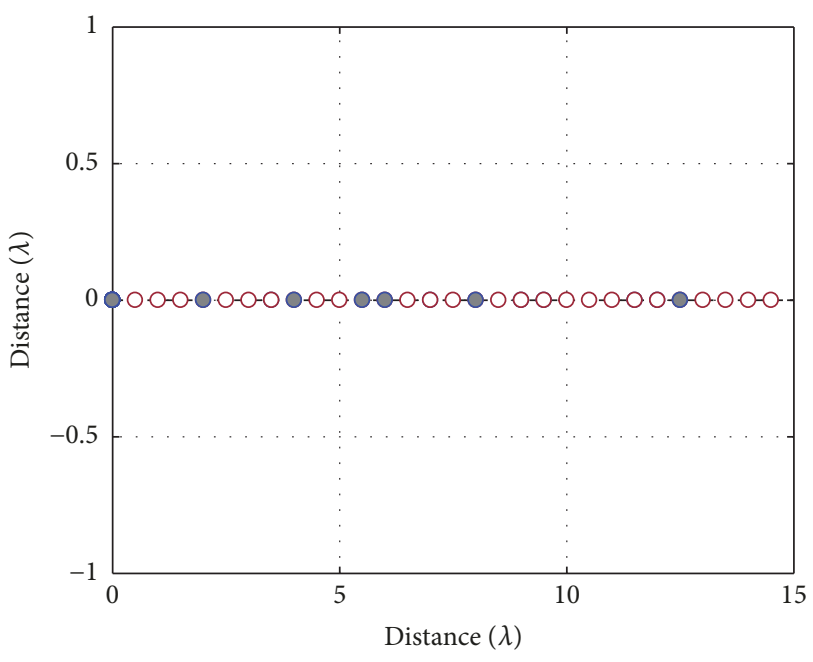

(a)

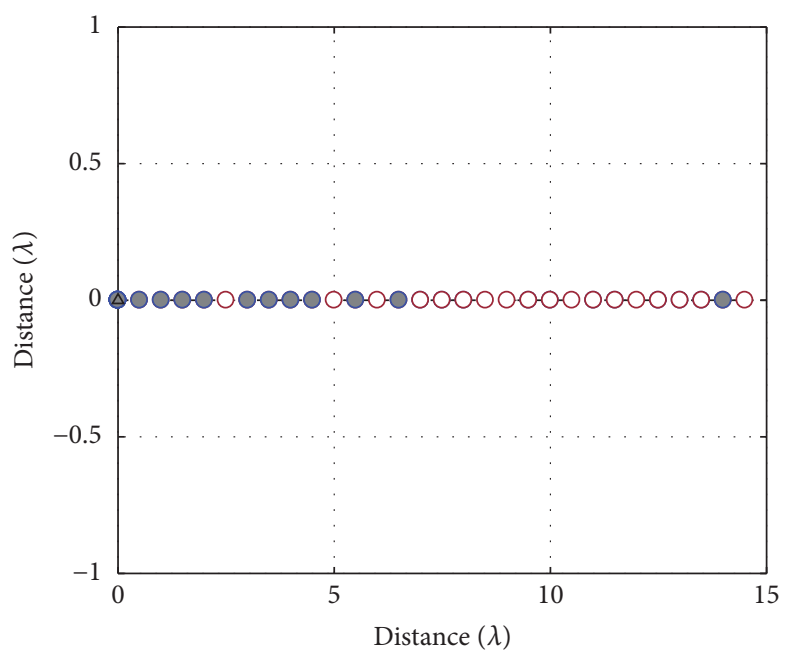

(b)

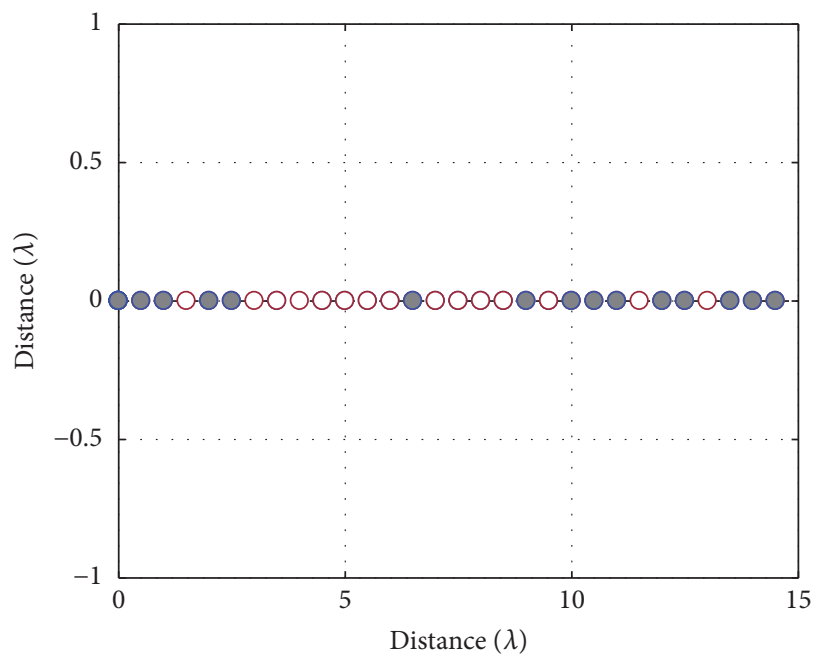

(c)

FIGURE 7: The sparsity of GNSS array obtained from employing the CSMC beamformer with different norm constraints: (a) $t=25 \% \times\left\|\mathbf{w}_{\text {opt }}\right\|_{1}$; (b) $t=45 \% \times\left\|\mathbf{w}_{\text {opt }}\right\|_{1}$; (c) $t=65 \% \times\left\|\mathbf{w}_{\text {opt }}\right\|_{1}$.

Figure 6 shows the detection probability versus SNR in the presence of impulsive noises. It can be observed from the results shown in Figure 6(a) that the proposed beamforming algorithm enjoys a higher detection probability than most of the conventional beamformers for the value of $\alpha=1.85$ which indicates lowly impulsive noises. As the value of $\alpha$ decreases in Figures 6(b) and 6(c), which means more highly impulsive noises exist, the proposed algorithms outperform the comparison methods significantly and achieve the highest rate of acquisition for satellite signal, whereas the detection probability of traditional algorithms is deteriorating and fluctuating in these two cases, which demonstrates that these methods are more sensitive to impulsive noises and fail to acquire GNSS signal in severely impulsive noises scenarios.

\subsection{The Sparsity of the ULA with CMSC in GNSS Receiver.} This section presents three sets of simulations carried out with a 30-element ULA (simulated large number of sensor array utilized in satellite communication system) to explore the sparsity of the proposed beamforming method for GNSS receiver. All the simulations were carried out using 3 distinct levels of norm constraint, according to Table 3. The elements represented by white circles are those turned off by the algorithm. The results depicted in Figure 7 indicate that the acquisition of satellite signal with ULA favors the sparse solution produced by CSMC algorithm, the GNSS beamformer synthesized by the CSMC with $25 \%, 45 \%$, and $65 \%$ norm constraints keeps only $16.67 \%, 26.67 \%$, and $40 \%$ of the total number of elements online, and the increasing sparsity was achieved decreasing the norm constraint value. From the results of simulation, we can conclude that the efficient ability of the proposed algorithm to produce sparse solutions and shut down a number of sensors in adaptive antenna arrays is suitable for GNSS receiver system.

4.6. The Influence of the Kernel Size and Step Parameter on CSMC Algorithm Performance. To evaluate the effects of all the used parameters on the behavior of the proposed 


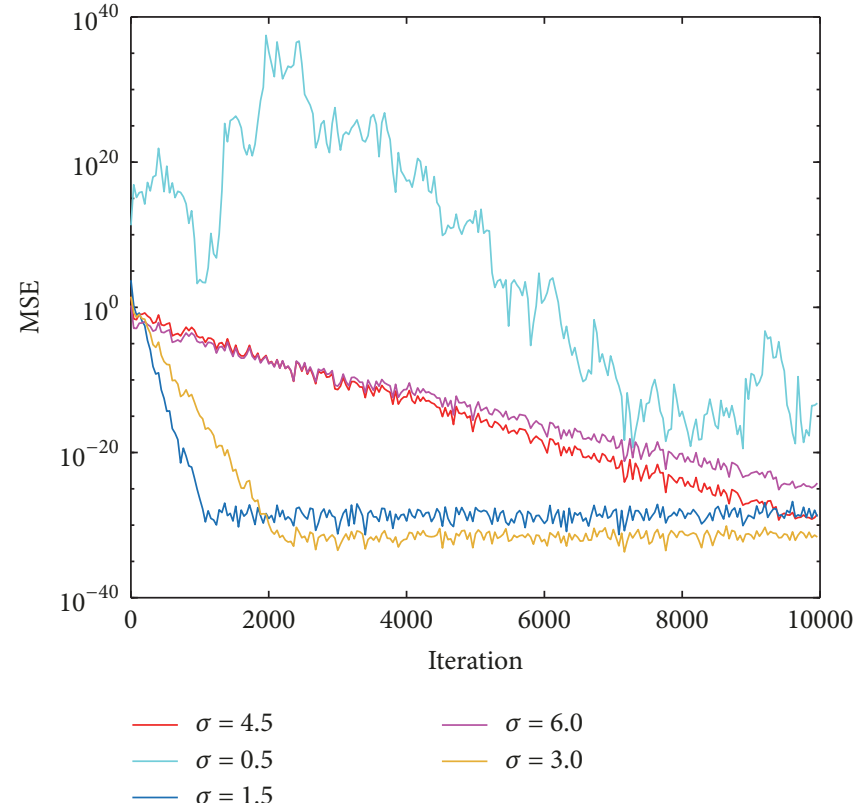

(a)

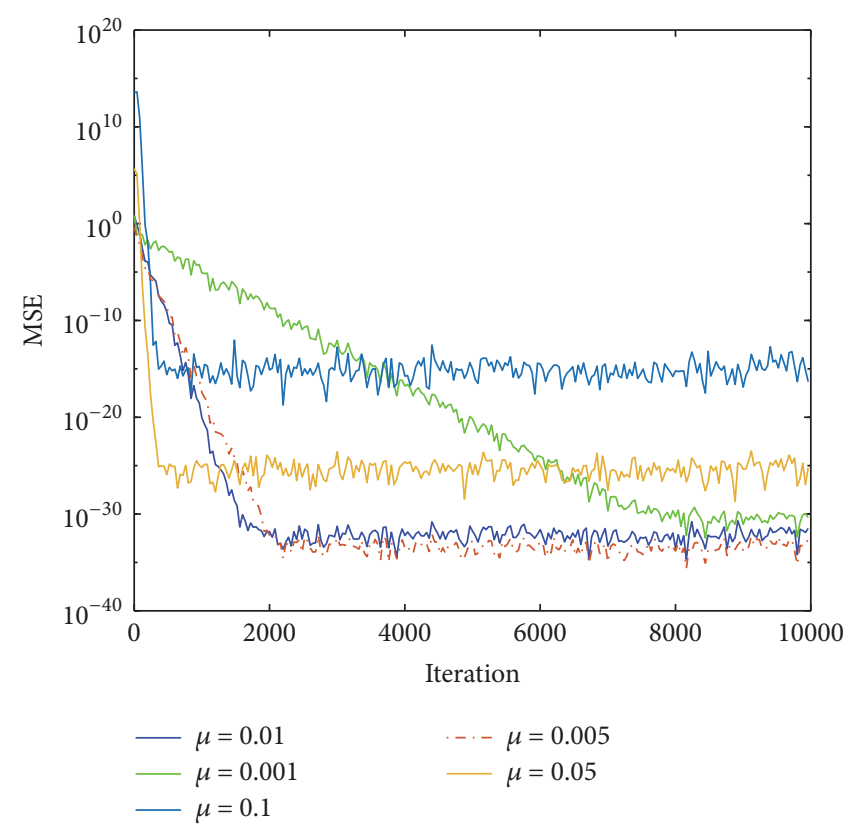

(b)

FIGURE 8: MSE learning curves of the proposed CSMC with different used parameters: (a) MSE for different kernel size; (b) MSE for different step parameter.

TABLE 3: Parameter values for sparsity simulations.

\begin{tabular}{lc}
\hline The Number of Elements & 30 \\
\hline L1 Norm Constraint $t$ & $t=\left\|\mathbf{w}_{\text {opt }}\right\|_{1}$ \\
\hline Norm Constraint Ratio & $25 \%, 45 \%, 65 \%$ \\
\hline
\end{tabular}

CSMC algorithm and fully justify these parameters and their influence on the results closely examined, we conduct a series of simulations in the context of adaptive beamforming, including simulations on kernel size and step parameter. The mean square error (MSE) measure between the local satellite signal replica and beamformer output is employed to quantify the performance in the literature. A total of 10000 iterations were performed for the verification. The simulation experiment environment of this example is the same as Section 4.1. In the first case, for investigating the effect of the kernel size on MSE with different level, we consider five sets of parameters to justify this parameter on our approach. Figure 8(a) illustrates the resultant MSE performance versus the number of iterations for different kernel sizes. It can be observed from the results shown in the figure that choosing a proper value for the kernel size $\sigma$ is a crucial issue in CSMC algorithm since it directly affects the adaptive learning speed and accuracy. $\sigma=0.5$ will lead to a very poor performance so that the kernel size cannot be chosen arbitrarily small. However, the large values of the kernel size $\sigma$ will lead to a low speed performance; thus, in this paper, we fix $\sigma=3$ in the simulation which benefits from both the high speed and the high accuracy, simultaneously. In the next case, we investigate the effects for different values of the step parameter on the CSMC performance. The MSE curve of simulation results for different $\mu$ is shown in Figure 8(b). As can be seen, the large step size leads to fast convergence rate, but the steady state MSE of the proposed algorithms increases as the step sizes increase, achieving higher steady state error, while small step size has small misadjustment for adaptive beamforming, but suffers from slow convergence speed. From the above, we can conclude that, to obtain performance improvement and stability with balanced convergence rate, we select $\mu=0.01$ to guarantee the fast convergence and satisfied stable steady state MSE for the proposed CSMC algorithm.

\section{Conclusion}

A novel adaptive beamforming approach is proposed aiming to improve the performance of GNSS signal acquisition in this paper. We incorporate the sparse norm regularization and constraint maximum correntropy criterion into the optimization constraint equation of beamforming to obtain a new adaptive beamformer which is able to mitigate the adverse effect of impulsive noises while allowing flexibility in the energy consumption at GNSS receiver. A detailed mean square analysis of the proposed methods was presented and the stability condition was derived. The numerical simulation results described in the paper indicate that the proposed CSMC beamformer provides better impulsive noises suppression for improved performance of satellite signal acquisition. Furthermore, with the sparsity produced by CSMC, a thinned 30 -element ULA is achieved to save energy to GNSS receiver. The proposed algorithms can also be extended to other array geometries and applications in GNSS signal processing. We plan to explore those applications in future work. 


\section{Data Availability}

The data used to support the findings of this study are available from the corresponding author upon request.

\section{Conflicts of Interest}

The authors declare that they have no conflicts of interest.

\section{References}

[1] S. A. Nik and M. G. Petovello, "Implementation of a dualfrequency GLONASS and GPS L1 C/A software receiver," Journal of Navigation, vol. 63, no. 2, pp. 269-287, 2010.

[2] M. J. Dow, R. E. Neilan, and C. Rizos, “The international GNSS service in a changing landscape of global navigation satellite system," Journal of Geodesy, vol. 83, no. 3-4, pp. 191-198, 2009.

[3] J. Arribas, C. Fernandez-Prades, and P. Closas, "Antenna array based GNSS signal acquisition for interference mitigation," IEEE Transactions on Aerospace and Electronic Systems, vol. 49, no. 1, pp. 223-243, 2013.

[4] O. Julien, C. Macabiau, M. E. Cannon, and G. Lachapelle, "Unambiguous sine-BOC (n,n) acquisition/tracking technique for navigation applications," IEEE Transactions on Aerospace and Electronic Systems, vol. 43, no. 1, pp. 150-162, 2007.

[5] D. Borio, "GNSS acquisition in the presence of continuous wave interference," IEEE Transactions on Aerospace and Electronic Systems, vol. 46, no. 1, pp. 47-60, 2010.

[6] Y. T. Morton, M. Miller, J. Tsui, D. Lin, and Q. Zhou, "GPS civil signal self-interference mitigation during weak signal acquisition," IEEE Transactions on Signal Processing, vol. 55, no. 12, pp. 5859-5863, 2007.

[7] G. X. Gao, M. Sgammini, M. Lu, and N. Kubo, "Protecting GNSS receivers from jamming and interference," Proceedings of the IEEE, vol. 104, no. 6, pp. 1327-1338, 2016.

[8] K. Sun, M. Zhang, and D. Yang, "A new interference detection method based on joint hybrid time-frequency distribution for GNSS receivers," IEEE Transactions on Vehicular Technology, vol. 65, no. 11, pp. 9057-9071, 2016.

[9] A. Broumandan, A. Jafarnia-Jahromi, S. Daneshmand, and G. Lachapelle, "Overview of spatial processing approaches for GNSS structural interference detection and mitigation," Proceedings of the IEEE, vol. 104, no. 6, pp. 1246-1257, 2016.

[10] W. Sun and M. Ainin, "Interference suppression for GPS coarse/acquisition signals using antenna array," in Proceedings of the 2004 IEEE International Conference on Acoustics, Speech, and Signal Processing, pp. 17-21, Montreal, Canada.

[11] L. W. Chen, J. S. Zheng, M. K. Su, and J. L. Zhang, "A strong interference suppressor for satellite signals in GNSS receivers," Circuits Systems Signal Processing, vol. 36, pp. 1-16, 2016.

[12] Z. Chen, H. Li, G. Cui, and M. Rangaswamy, "Adaptive transmit and receive beamforming for interference mitigation," IEEE Signal Processing Letters, vol. 21, no. 2, pp. 235-239, 2014.

[13] D. T. M. Slock, "On the Convergence Behavior of the LMS and the Normalized LMS Algorithms," IEEE Transactions on Signal Processing, vol. 41, no. 9, pp. 2811-2825, 1993.

[14] J. Kim and A. Poularikas, "Performance analysis of the adjusted step size nlms algorithm," in Proceedings of the Thirty-Sixth Southeastern Symposium on System Theory, 2004., pp. 467-471, Atlanta, GA, USA.
[15] L. C. Godara and A. Cantoni, "Analysis of constrained LMS algorithm with application to adaptive beamforming using perturbation sequences," IEEE Transactions on Antennas and Propagation, vol. 34, no. 3, pp. 368-379, 1986.

[16] M. Dosaranian-Moghadam, H. R. Bakhshi, G. R. Dadashzadeh, and P. Rahmati, "Adaptive beamforming method based on constrained LMS algorithm for tracking mobile user," in Proceedings of the 2009 Global Mobile Congress, GMC 2009, pp. 12 14, IEEE, China, October 2009.

[17] H. C. Woo, "Variable step size LMS algorithm using squared error and autocorrelation of error," Procedia Engineering, vol. 41, pp. 47-52, 2012.

[18] R. He, B. Hu, W. Zheng, and X. Kong, "Robust principal component analysis based on maximum correntropy criterion," IEEE Transactions on Image Processing, vol. 20, no. 6, pp. 14851494, 2011.

[19] Y. Li, Y. Wang, R. Yang, and F. Albu, "A soft parameter function penalized normalized maximum correntropy criterion algorithm for sparse system identification," Entropy, vol. 19, no. 1, 2017.

[20] R. He, W.-S. Zheng, and B.-G. Hu, "Maximum correntropy criterion for robust face recognition," IEEE Transactions on Pattern Analysis and Machine Intelligence, vol. 33, no. 8, pp. 15611576, 2011.

[21] W. Wang, Q. Du, R. Wu, and J. Liang, "Interference suppression with flat gain constraint for satellite navigation systems," IET Radar, Sonar \& Navigation, vol. 9, no. 7, pp. 852-856, 2015.

[22] J. F. De-Andrade, M. L. R. De-Campos, and J. A. Apolinario, "An L1-norm linearly constrained LMS algorithm applied to adaptive beamforming," Sensor Array \& Multichannel Signal Processing Workshop, pp. 17-20, 2012.

[23] K. Shi and X. Ma, "A variable-step-size NLMS algorithm using statistics of channel response," Signal Processing, vol. 90, no. 6, pp. 2107-2111, 2010.

[24] W. Ma, H. Qu, G. Gui, L. Xu, J. Zhao, and B. Chen, "Maximum correntropy criterion based sparse adaptive filtering algorithms for robust channel estimation under non-Gaussian environments," Journal of The Franklin Institute, vol. 352, no. 7, pp. 27082727, 2015.

[25] H. Zhou, "On some trace inequalities for positive definite Hermitian matrices," Journal of Inequalities and Applications, $\mathrm{p}$. 64, 2014

[26] W. Gabriel, "Preface-Special issue on adaptive antennas," IEEE Trans. Antennas \& Propagation, vol. 24, pp. 573-574, 1976.

[27] F. Chen, J. Nie, S. Ni, Z. Li, and F. Wang, "Combined algorithm for interference suppression and signal acquisition in GNSS receivers," IEEE Electronics Letters, vol. 53, no. 4, pp. 274-275, 2017.

[28] P. Misra, W. Hu, Y. Jin et al., "Energy efficient GPS acquisition with Sparse-GPS," in Proceedings of the 13th ACM/IEEE International Conference on Information Processing in Sensor Networks (IPSN), pp. 15-17, April 2014. 


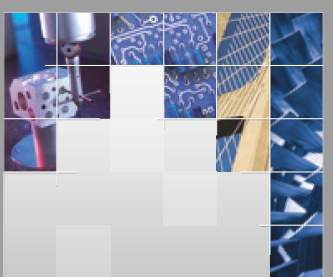

\section{Enfincering}
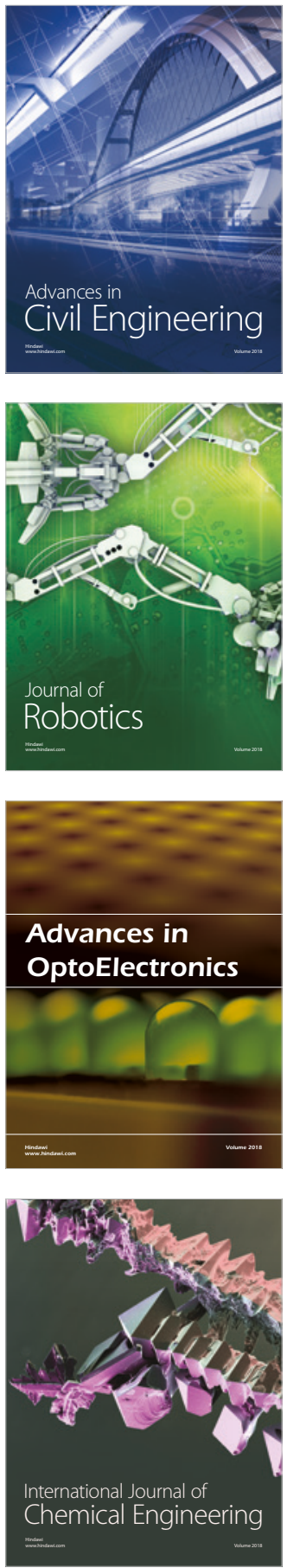

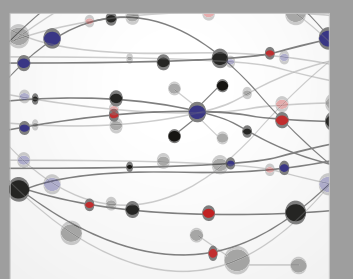

\section{Rotating \\ Machinery}

The Scientific World Journal

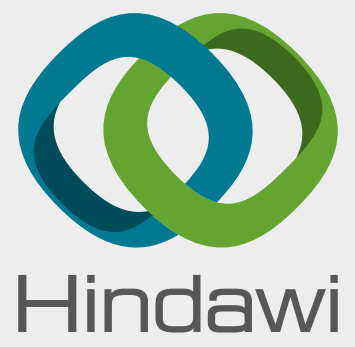

Submit your manuscripts at

www.hindawi.com
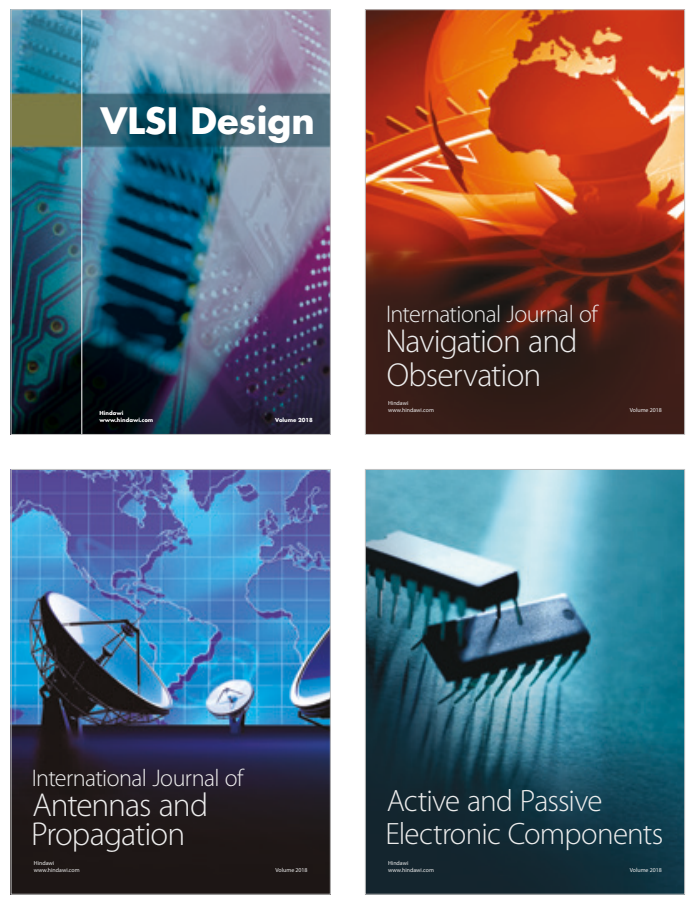
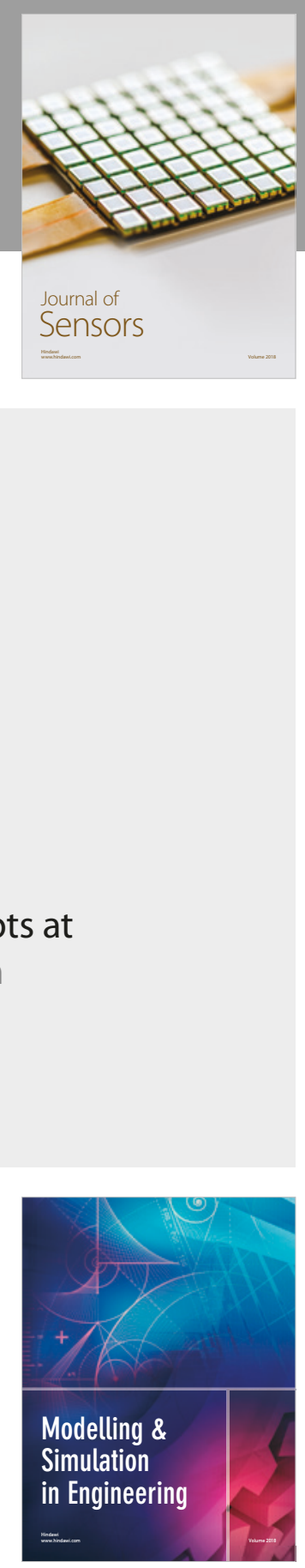

\section{Advances \\ Multimedia}
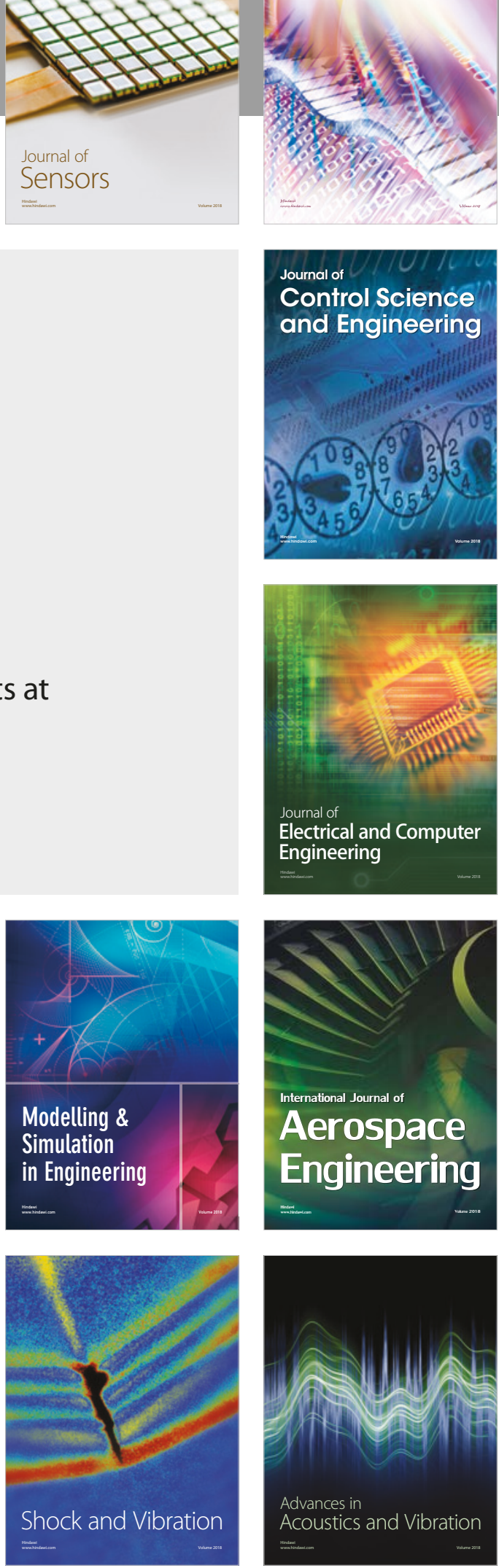